\title{
An overview of $J / \psi$ production in heavy ion collisions at PHENIX
}

\author{
Matthew Wysocki ${ }^{\mathrm{a}}$ \\ for the PHENIX Collaboration \\ Department of Physics, University of Colorado, Boulder, CO 80309-0390, USA
}

Received: 1 October 2008 / Revised: 5 March 2009 / Published online: 21 March 2009

(C) Springer-Verlag / Società Italiana di Fisica 2009

\begin{abstract}
This is an overview of the PHENIX $J / \psi$ results in hot nuclear matter from heavy ion collisions. Current results for $R_{\mathrm{AA}}$ and $v_{2}$ in $\mathrm{Au}+\mathrm{Au}$ collisions, as well as $R_{\mathrm{AA}}$ from $\mathrm{Cu}+\mathrm{Cu}$ collisions are included and discussed. A comparison is also done to cold nuclear matter effects using $R_{\mathrm{dAu}}$ results.
\end{abstract}

PACS 25.75.-q $\cdot$ 12.38.Mh $\cdot 13.20 . \mathrm{Gd}$

\section{Introduction}

The Relativistic Heavy Ion Collider (RHIC) at Brookhaven National Laboratory creates a hot, dense medium using nuclear collisions at high center-of-mass energies. It is expected that the medium undergoes a phase transition from normal nuclear matter to a Quark-Gluon Plasma (QGP), a color-charge plasma of (locally) deconfined quarks and gluons.

$J / \psi$ suppression has long been expected to be an important signature of the formation of the QGP [1]. Since its proposal, there have been many further developments in our understanding of competing effects. We expect a significant suppression due to cold nuclear matter (CNM) effects such as nuclear shadowing and gluon saturation [2]. There are then further complications such as feed-down from heavier charmonium states [3] and recombination of $c \bar{c}$ pairs [4].

\section{The PHENIX experiment}

PHENIX measures $J / \psi \mathrm{s}$ through two channels. At midrapidities $(|y|<0.35), J / \psi \rightarrow \mathrm{e}^{+} \mathrm{e}^{-}$is measured by the central arm detectors, which include the Electromagnetic Calorimeter, Drift Chamber, Pad Chamber and Ring-

a e-mail: wysockim@ colorado.edu
Imaging Cerenkov Counters. At backward and forward rapidities $(1.2<|y|<2.2) J / \psi \rightarrow \mu^{+} \mu^{-}$is measured by the muon arm detectors, which include the Muon Tracker and Muon Identifier.

In addition, the Beam-Beam Counter (BBC, $3<|\eta|<$ 3.9) is used to measure collision centrality and vertex, and the Reaction Plane Detector (RXNP) was installed and used to measure the reaction plane of the collision starting with the $2007 \mathrm{Au}+\mathrm{Au}$ run. For more detail on the PHENIX detectors, see [5].

\section{Nuclear modification factor $\boldsymbol{R}_{\mathrm{AA}}$}

The nuclear modification factor $R_{\mathrm{AA}}$ (see (1)) quantifies the suppression or enhancement of particle production in collisions of heavier nuclei with respect to $\mathrm{p}+\mathrm{p}$ collisions, scaled by the appropriate number of binary collisions $\left\langle N_{\text {coll }}\right\rangle$ in the heavier species, typically as calculated by a Glauber model [6]. Therefore, an $R_{\mathrm{AA}}=1$ would indicate that the nuclear collisions can be treated as a simple collection of independent nucleon-nucleon collisions, while deviations from unity indicate that more complicated physics is involved.

$R_{\mathrm{AA}}=\frac{1}{\left\langle N_{\mathrm{coll}}\right\rangle} \frac{d N_{J / \psi}^{\mathrm{Au}+\mathrm{Au}} / d y}{d N_{J / \psi}^{\mathrm{p}+\mathrm{p}} / d y}$.

The values of $R_{\mathrm{AA}}$ presented here are from $\mathrm{Au}+\mathrm{Au}$ data taken during 2004 [7] and $\mathrm{Cu}+\mathrm{Cu}$ in 2005 [8], with the denominator coming from $2005 \mathrm{p}+\mathrm{p}$ collisions [9] in all cases, and all collisions at $\sqrt{s_{N N}}=200 \mathrm{GeV}$.

In the top portion of Fig. 1, $R_{\mathrm{AA}}$ is plotted for the 2004 $\mathrm{Au}+\mathrm{Au}$ data as a function of the number of participant nucleons $N_{\text {part }}$ in the collision, both at forward and midrapidity [7]. The uncertainties are broken into three categories for all data plots: the statistical and uncorrelated systematic uncertainties are represented by the error bars, the 


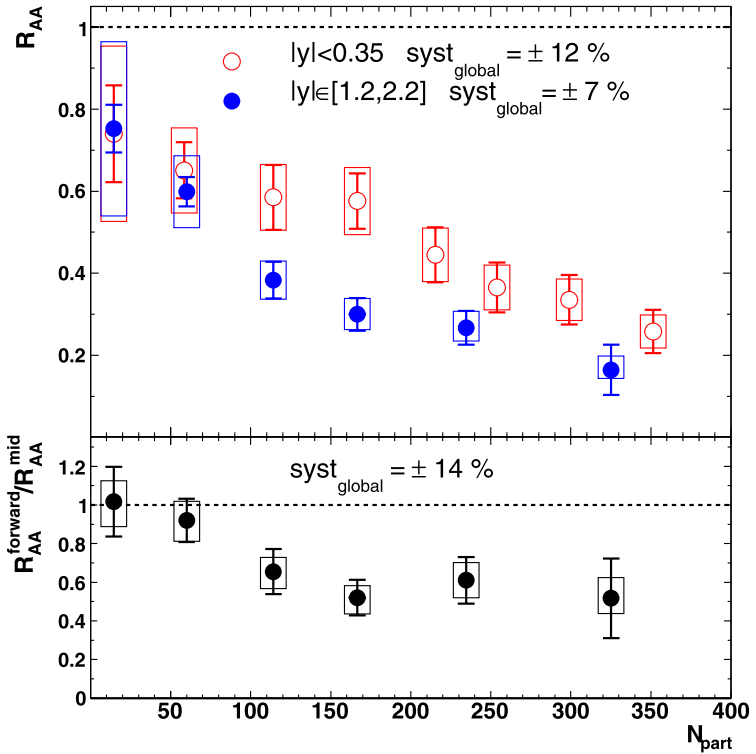

Fig. 1 (Color online) Top: $R_{\mathrm{AA}}$ for the $2004 \mathrm{Au}+\mathrm{Au}$ as a function of $N_{\text {part }}$ at mid-rapidity (open red circles) and forward rapidity (closed blue circles) [7]. Bottom: ratio of forward to mid-rapidity $R_{\mathrm{AA}}$ as a function of $N_{\text {part }}$

systematic uncertainties that are correlated point-to-point are represented in the boxes, and the overall normalization uncertainty is quoted in each figure.

As can be seen in Fig. $1, J / \psi$ production is suppressed in more central collisions (larger $N_{\text {part }}$ ). In addition, production is more suppressed at forward rapidity than at mid-rapidity. To make this clear, the ratio of the two rapidity bins is shown in the bottom half of the Figure.

This runs contrary to what one might expect in a simple suppression model based on local energy density, which would be higher at mid-rapidity and lead to the opposite trend in the ratio of Fig. 1. On the other hand, recombination of $c \bar{c} \rightarrow J / \psi$ in the medium would reduce the level of suppression, and this effect is expected to be larger at midrapidity due to the peak there in the $c$-quark distribution.

In Figure 2 the $\mathrm{Au}+\mathrm{Au} R_{\mathrm{AA}}$ is plotted as a function of $p_{T}$ in 4 centrality bins. There has been some interest recently $[10,11]$ as to whether the $J / \psi R_{\mathrm{AA}}$ approaches unity at higher $p_{T}$ or remains suppressed. There is a rising trend in $R_{\mathrm{AA}}$ in the $\mathrm{Au}+\mathrm{Au} 20-40 \%$ and $40-60 \%$ centrality bins; unfortunately, as can be seen in the figure, PHENIX is currently limited by statistics to $p_{T} \sim 5 \mathrm{GeV} / \mathrm{c}$. It is hoped that the $2007 \mathrm{Au}+\mathrm{Au}$ data can extend this range slightly.

One model that has drawn interest to the $p_{T}$ spectrum is the so-called "Hot Wind" model [12], which uses the AdS/CFT correspondence to model the velocity-dependence of the screening length for heavy quarkonia such as the $J / \psi$. As a consequence it predicts that the $J / \psi$ dissociation temperature decreases with increasing $J / \psi p_{T}$. Experimentally, if the medium produced has a temperature slightly below

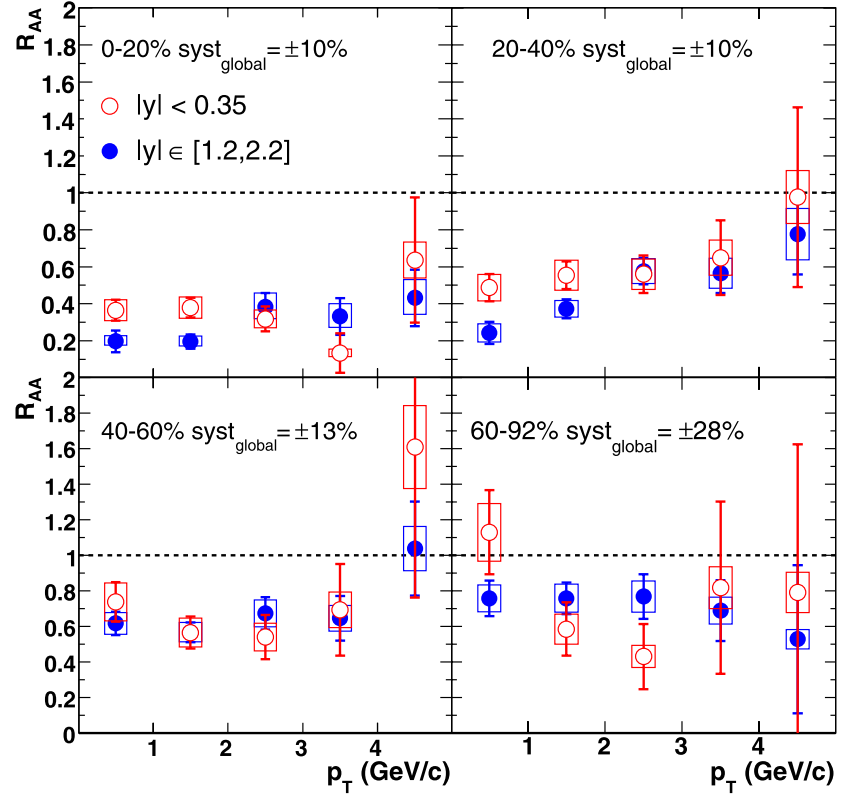

Fig. 2 (Color online) $R_{\mathrm{AA}}$ for the $2004 \mathrm{Au}+\mathrm{Au}$ as a function of transverse momentum in four different bins of centrality $(0-20 \%, 20-40 \%$, $40-60 \%$, and $60-92 \%$ ). Mid-rapidity is represented by open red circles, while forward rapidity is the closed blue circles [7]

the dissociation temperature for low- $p_{T} J / \psi \mathrm{s}$, but slightly above for high- $p_{T} J / \psi$ s, this could appear as a cutoff in $p_{T}$ where the $J / \psi$ suppression quickly turns on. Let us refer to this quick turn-on point as $p_{T}$ (transition). Since the medium temperature is expected to increase as a function of collision centrality, given a certain set of temperatures, one could in principle observe this $p_{T}$ (transition) decrease as one goes to more central events. It is notable that if, for a given centrality, the medium temperature is higher than the dissociation temperature for even the lowest- $p_{T} J / \psi \mathrm{s}$, then no quick turn-on versus $p_{T}$ will be present. The same would be true if the highest- $p_{T} J / \psi \mathrm{s}$ in the measurement range have a dissociation temperature below the medium temperature. However, this does not include other effects such as recombination, which complicate this picture and make quantitative comparisons more difficult.

$J / \psi R_{\mathrm{AA}}$ has also been measured in $\mathrm{Cu}+\mathrm{Cu}$ collisions during 2005, and is plotted vs. $N_{\text {part }}$ in Fig. 3 . The values are consistent with their $\mathrm{Au}+\mathrm{Au}$ counterparts within the error bars over their common range of $N_{\text {part }}$.

In interpreting these results, it is important to quantify the CNM effects in order to see how much of the suppression is actually due to the hot nuclear medium. In [2] we calculate the $R_{\mathrm{dAu}}$ (identically to the $R_{\mathrm{AA}}$ above) for the $2003 \mathrm{~d}+\mathrm{Au}$ and $2005 \mathrm{p}+\mathrm{p}$ datasets. We then fit a simple model of a breakup cross section ( $\left.\sigma_{\text {breakup }}\right)$ for the $J / \psi$ in the nucleus combined with a nuclear-modified PDF such as EKS [13] or NDSG [14]. The fit takes into account all of the statistical and systematic errors associated with the data, and we ex- 


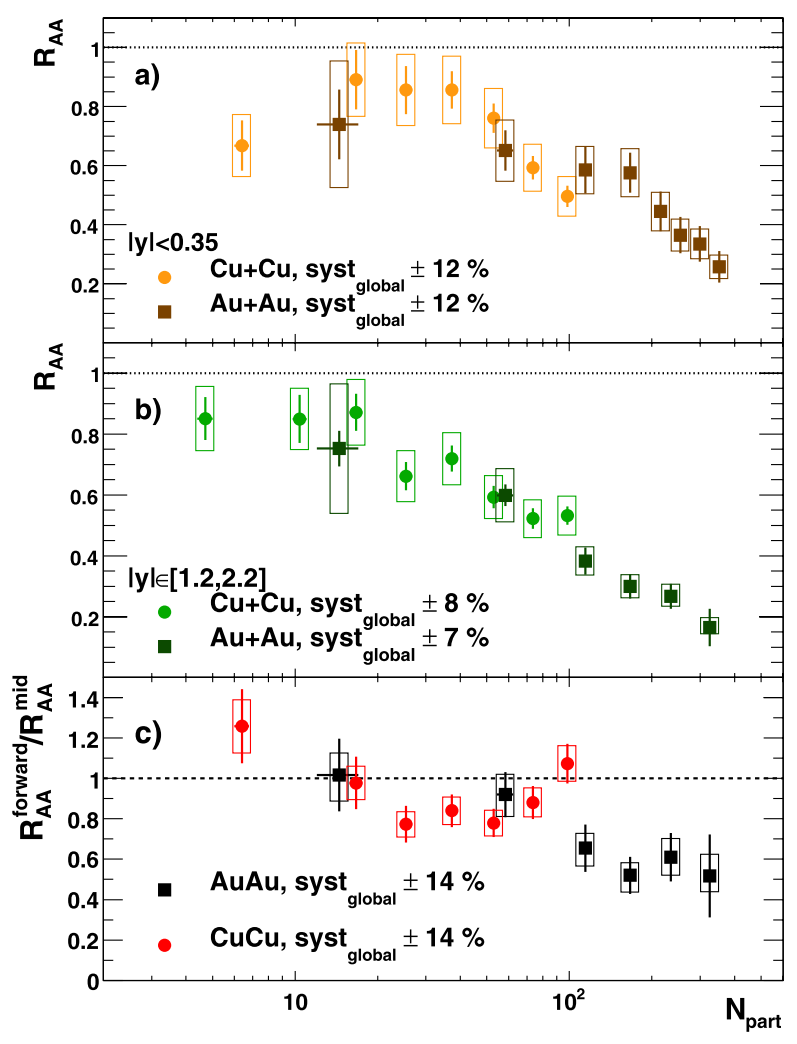

Fig. 3 (Color online) $R_{\mathrm{AA}}$ for the $2005 \mathrm{Cu}+\mathrm{Cu}$ (circles) and the 2004 $\mathrm{Au}+\mathrm{Au}$ (squares) collisions as a function of $N_{\text {part }}$ at mid-rapidity (a), forward rapidity (b), and their ratio (c) [8]

tract values of $\sigma_{\text {breakup }}=2.8_{-1.4}^{+1.7} \mathrm{mb}$ and $\sigma_{\text {breakup }}=2.2_{-1.5}^{+1.6}$ mb using EKS and NDSG, respectively. These fits are shown in Fig. 4 for the EKS model, where the yellow band represents the best fit \pm one standard deviation.

These break-up cross sections, extracted from d + Au collisions where only CNM effects are present, can then be used to make quantitative predictions for the CNM effects in $\mathrm{Au}+\mathrm{Au}$ collisions. Treating the $\mathrm{d}+\mathrm{Au}$ fits as the result of a nucleon + Au collision, we then apply a model of the impact parameter-dependence of the nuclear PDFs [15,

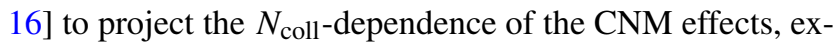
tend the projection of CNM effects all the way to the central $\mathrm{Au}+\mathrm{Au}$ collisions.

These predictions are shown in Fig. 5, overlayed atop the $\mathrm{Au}+\mathrm{Au}$ data points. The bands represent the 1-standard deviation error due to the combined statistical and systematic uncertainties in the $\mathrm{d}+\mathrm{Au}$ data. They do not include any uncertainty due to the theoretical model. As can be seen in the figures, the forward rapidity $\mathrm{Au}+\mathrm{Au}$ data is suppressed beyond the level of CNM projections, but at mid-rapidity it is only the most central points that are significantly suppressed. It should be noted that the large uncertainties make any definitive statements difficult, and in addition, the error bands are correlated between the two rapidities as they come from the same fit.
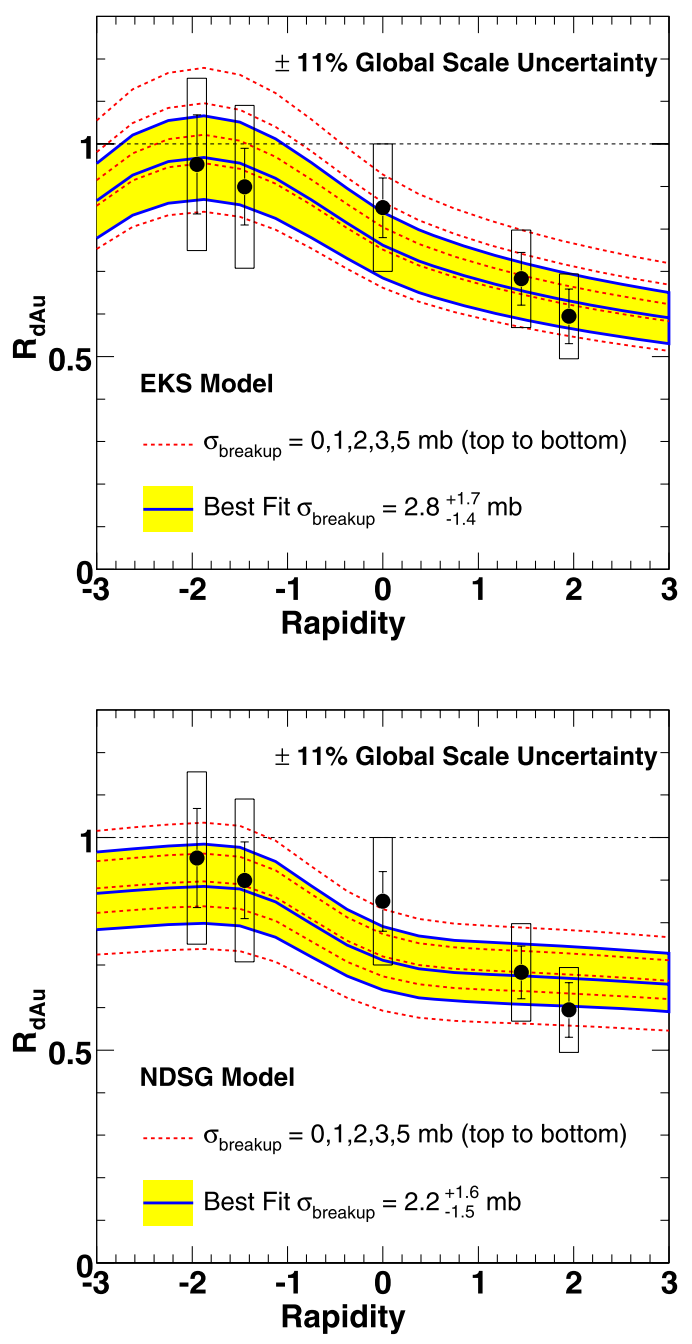

Fig. 4 (Color online) $R_{\mathrm{dAu}}$ data compared to various theoretical

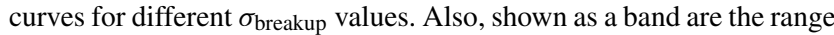
of $\sigma_{\text {breakup }}$ found to be consistent with the data within one standard deviation. The top panel is a comparison for EKS shadowing [13], while the bottom panel is for NDSG shadowing [14]. Taken from [2]

The $2008 \mathrm{~d}+\mathrm{Au}$ dataset includes about 30 times the integrated luminosity of the 2003 data, and is currently being analyzed. We are hopeful that it will provide much a better constraint on the CNM effects on the $J / \psi$ in $\mathrm{Au}+\mathrm{Au}$ collisions.

\section{$4 J / \psi$ elliptic flow}

Another topic of great interest is the flow of heavy quarks. We measure elliptic flow using the second Fourier coefficient in $\phi, v_{2}$ :

$v_{2}=\langle\cos 2(\phi-\Psi)\rangle$

where $\phi$ is the azimuthal angle of the particle of interest and $\Psi$ is the reaction plane of the collision. 

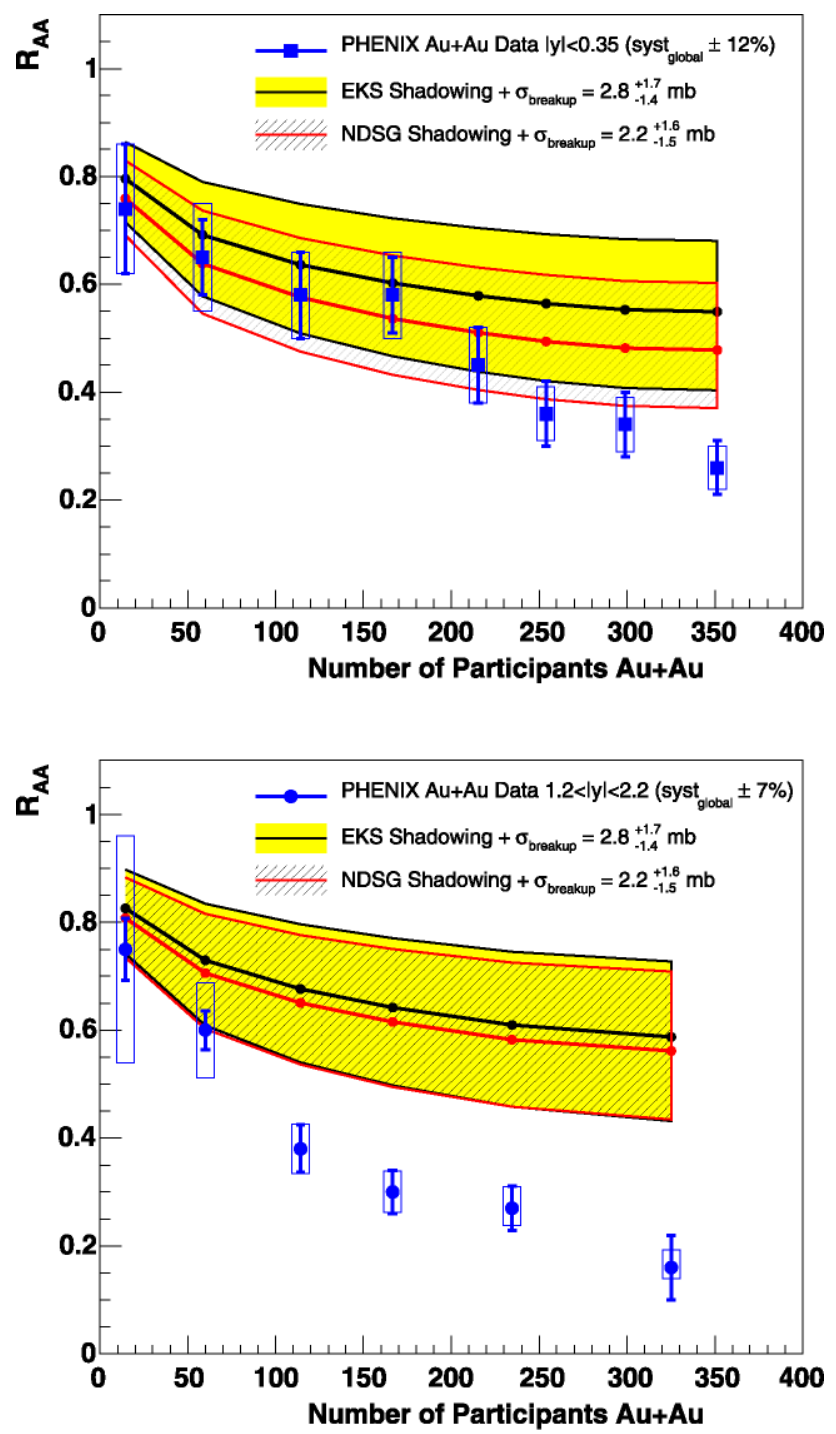

Fig. 5 (Color online) $R_{\mathrm{AA}}$ for $\mathrm{Au}+\mathrm{Au}$ [7] collisions compared to a band of theoretical curves for the $\sigma_{\text {breakup }}$ values found to be consistent with the $\mathrm{d}+\mathrm{Au}$ data as shown in Fig. 4. The top figure includes both EKS shadowing [13] and NDSG shadowing [14] at mid-rapidity. The bottom figure is the same at forward rapidity

Because cosine is an even function, fluctuations in the reaction plane measurement lead to a bias in the measured $v_{2}$ which must be corrected by the factor:

$v_{2}^{\text {true }}=v_{2}^{\text {meas }} /\left\langle\cos 2\left(\Psi^{\text {true }}-\Psi^{\text {meas }}\right)\right\rangle$.

This correction factor is plotted in Fig. 6 as a function of collision centrality. It is reduced by almost a factor of two by the Reaction Plane Detector, as compared to measurements using the BBC [18], simply due to the higher particle multiplicity in the rapidity coverage of the Reaction Plane Detector.

Precise measurements of $J / \psi v_{2}$ could give us insight into how well they thermalize with the medium, as well as how big an effect recombination is. Since we would expect

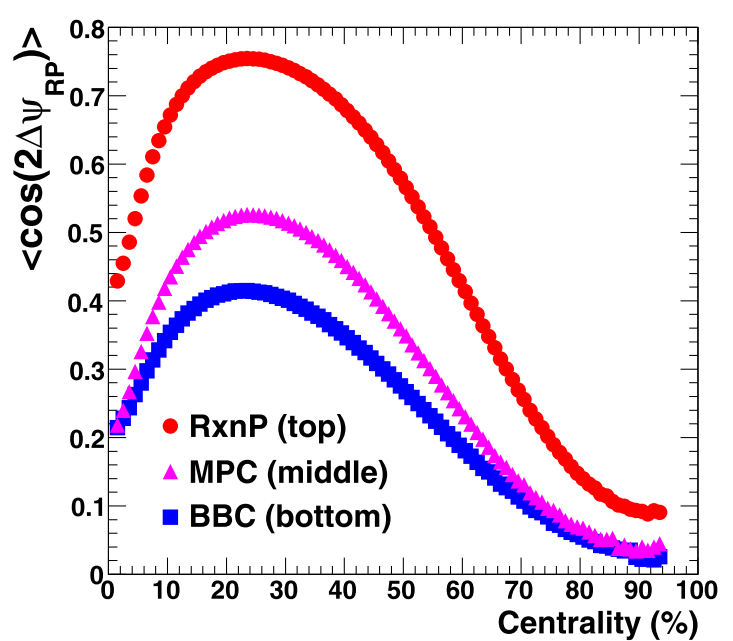

Fig. 6 Reaction plane resolution correction factor (see (3)) in 2007 $\mathrm{Au}+\mathrm{Au}$ collisions as a function of centrality for several PHENIX detectors [18]

$J / \psi$ s that form via recombination to inherit the flow properties of their constituent quarks, and we know from previous measurements [17] that open charm mesons have a large $v_{2}$, we would then expect that $J / \psi$ s from recombination would also exhibit a large $v_{2}$. This could also manifest as a difference in $J / \psi v_{2}$ between forward and mid-rapidity, due to different contributions from recombination.

To this end, PHENIX has measured $J / \psi v_{2}$ in $\sqrt{s_{N N}}=$ $200 \mathrm{GeV} \mathrm{Au}+\mathrm{Au}$ collisions for the first time in 2007, making use of the new Reaction Plane Detector [18]. $v_{2}$ as a function of $p_{T}$ is shown in Fig. 7 for both $J / \psi \rightarrow \mathrm{e}^{+} \mathrm{e}^{-}$ and $J / \psi \rightarrow \mu^{+} \mu^{-}$.

Both measurements are more consistent with zero or a negative $v_{2}$ than with a positive $v_{2}$. As can be seen in the Figure, though, the error bars remain much too large to offer any constraint on the available models. This represents only $42 \%$ of the data for the dielectron measurement, so there is still some improvement to be had.

The dimuon measurement, however, included roughly $90 \%$ of the data that is available. Some improvement is still possible by analyzing the minimum-bias data instead of the filtered sample that was used, as well as using event mixing instead of like-sign subtraction to estimate the combinatoric background, which is a more significant problem in the Muon Arms. A reduction by $\sqrt{2}$ is feasible, but that would still not be enough to discriminate between the available models.

\section{Summary}

We have presented $J / \psi R_{\mathrm{AA}}$ as a function of $N_{\text {part }}$ and $p_{T}$ in $\sqrt{s_{N N}}=200 \mathrm{GeV} \mathrm{Cu}+\mathrm{Cu}$ and $\mathrm{Au}+\mathrm{Au}$ collisions. It 
Fig. 7 (Color online) $J / \psi v_{2}$ in the $2007 \mathrm{Au}+\mathrm{Au}$ collisions as a function of transverse momentum at mid-rapidity (open blue circles) and forward rapidity (closed magenta circles). See [18] for details of the measurement and the comparison to various models

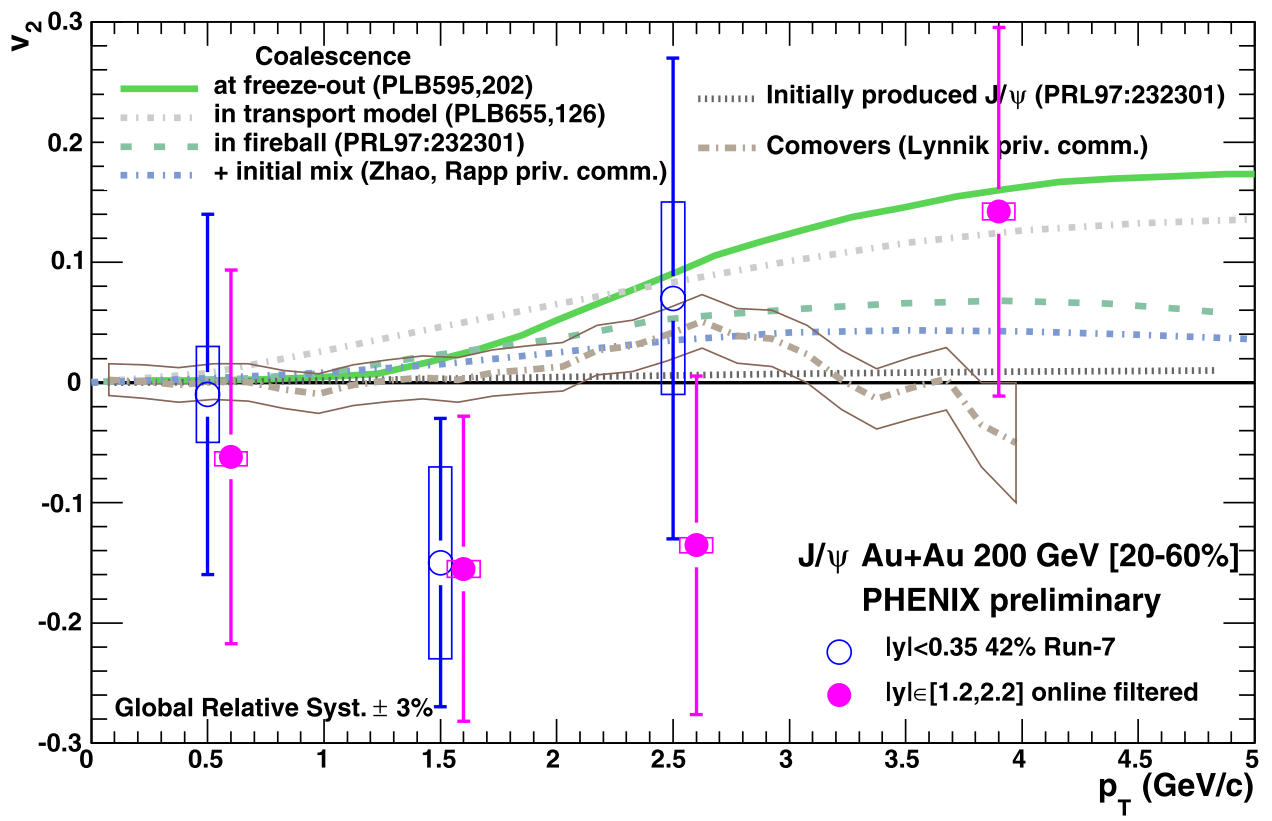

was found that $J / \psi$ production is more suppressed at forward rapidity than mid-rapidity in $\mathrm{Au}+\mathrm{Au}$ collisions, and that the suppression is comparable between $\mathrm{Au}+\mathrm{Au}$ and $\mathrm{Cu}+\mathrm{Cu}$ collisions of the same average values of $N_{\text {part }} . J / \psi$ suppression as a function of $p_{T}$ in $\mathrm{Au}+\mathrm{Au}$ collisions was found to be flat or rising with $p_{T}$, with large uncertainties at $p_{T}>4 \mathrm{GeV} / c$.

$J / \psi R_{\mathrm{dAu}}$ results have been combined with two nuclearmodified PDFs, used to make estimates of the $J / \psi$ breakup cross section within the nucleus, and project these effects to $\mathrm{Au}+\mathrm{Au}$ collisions. Statistically significant suppression of $J / \psi$ s was found to occur at forward rapidity and in the most central bin of mid-rapidity. However, the large uncertainties (which are correlated between forward and mid-rapidity) should be noted. The $2008 \mathrm{~d}+$ Au data is currently being analyzed, and includes over an order of magnitude increase in statistics.

We have also presented the first PHENIX measurements of $J / \psi v_{2}$ in $\mathrm{Au}+\mathrm{Au}$ collisions as a function of $p_{T}$, and compared them to several theoretical calculations. Both the forward and mid-rapidity points are more compatible with zero or negative $v_{2}$, but the large error bars discourage any quantitative comparisons at this point. Therefore, in order to provide quantitative constraints, a much larger $J / \psi$ sample will be required. This most likely will not happen until the RHIC II luminosity upgrades.

Acknowledgements M.G.W. acknowledges funding from the Division of Nuclear Physics of the U.S. Department of Energy under Grant No. DE-FG02-00ER41152.

\section{References}

1. T. Matsui, H. Satz, Phys. Lett. B 178, 416 (1986)

2. A. Adare et al. (PHENIX Collaboration), Phys. Rev. C 77, 024912 (2008). arXiv:0711.3917

3. F. Karsch, D. Kharzeev, H. Satz, Phys. Lett. B 637, 75 (2006)

4. L. Grandchamp, R. Rapp, G.E. Brown, Phys. Rev. Lett. 92, 212301 (2004)

5. K. Adcox et al. (PHENIX Collaboration), Nucl. Instrum. Methods A 499, 469 (2003)

6. M.L. Miller, K. Reygers, S.J. Sanders, P. Steinberg, Annu. Rev. Nucl. Part. Sci. 57, 205 (2007). arXiv:nucl-ex/0701025

7. A. Adare et al. (PHENIX Collaboration), Phys. Rev. Lett. 98, 232301 (2007). arXiv:nucl-ex/0611020

8. A. Adare et al. (PHENIX Collaboration), Phys. Rev. Lett. 101, 122301 (2008). arXiv:0801.0220

9. A. Adare et al. (PHENIX Collaboration), Phys. Rev. Lett. 98, 232002 (2007). arXiv:hep-ex/0611020

10. X. Zhao, R. Rapp, arXiv:0806.1239

11. Z. Tang (STAR Collaboration), J. Phys. G: Nucl. Part. Phys. 35, 104135 (2008). arXiv:0804.4846

12. H. Liu, K. Rajagopal, U. Wiedemann, Phys. Rev. Lett. 98, 182301 (2007). arXiv:hep-ph/0607062v3

13. K.J. Eskola, V.J. Kolhinen, R. Vogt, Nucl. Phys. A 696, 729 (2001). arXiv:hep-ph/0104124

14. D. deFlorian, R. Sassot, Phys. Rev. D 69, 074028 (2004). arXiv:hep-ph/0311227

15. S.R. Klein, R. Vogt, Phys. Rev. Lett. 91, 142301 (2003). arXiv:nucl-th/0305046

16. R. Vogt, Phys. Rev. C 71, 054902 (2005). arXiv:hep-ph/0411378

17. A. Adare et al. (PHENIX Collaboration), Phys. Rev. Lett. 98, 172301 (2007). arXiv:nucl-ex/0611018

18. C. Silvestre (PHENIX Collaboration), arXiv:0808.2925 